\title{
Orbitozygomatic craniotomy and trans-sylvian approach for resection of a tuberculum sella meningioma with extension to the posterior fossa
}

\author{
Georgios Klironomos, MD, PhD, and Amir R. Dehdashti, MD
}

Department of Neurosurgery, Hofstra Northwell School of Medicine, North Shore University Hospital, Manhasset, New York

Tuberculum sella meningiomas can be approached through lateral approaches including pterional/orbitozygomatic craniotomies, frontobasal craniotomy, or through expanded endoscopic transsphenoidal approaches. The authors present the case of a 60 -year-old male who presented with bitemporal hemianopia and significant right-side visual acuity compromise due to a large tuberculum sella meningioma. The tumor had an important extension to the posterior fossa. A right orbitozygomatic trans-sylvian approach was deemed most suitable to tackle the posterior extension of the tumor. Some operative nuances are detailed in the video including dissection of the tumor off the carotid artery, basilar bifurcation, and small thalamoperforators attached to the tumor. Postoperatively, the patient's bilateral hemianopia improved significantly, but his right visual activity remained unchanged. The remaining part of the tumor in the sella and midclivus was addressed with a second-stage expanded endoscopic transclival approach.

The video can be found here: https://youtu.be/KbewhlT2FWs.

KEYWORDS orbitozygomatic craniotomy; tuberculum sella meningioma 